\title{
Perspectives on the draft ICH-M10 guidance: an interview with Boris Gorovits
}

\author{
Boris Gorovits*,1 \\ ${ }^{1}$ Pfizer Inc., 1 Burtt Rd., Andover MA 01810, USA \\ *Author for correspondence: boris.gorovits@pfizer.com
}

\begin{abstract}
Biography: Boris Gorovits is a Senior Director of the Bioanalytical lab at Pfizer. Boris earned his PhD in Enzymology from the Moscow State University and later completed postdoctoral research studies in protein biophysics at the Medical Center, University of Texas at San Antonio, TX, USA. In 2000, Boris joined Wyeth Research (later Pfizer Inc) to work as a bioanalytical group lead with a growing scope of responsibilities. Currently, he leads the Bioanalytical group within Biomedicine Design department which is responsible for many aspects of the Regulated and Nonregulated Bioanalytical support for the pan-Pfizer Biotherapeutic portfolio. Boris co-chairs Pfizer internal Immunogenicity Expert Working Group, which is responsible for review of the biotherapeutic immunogenicity risk assessment and mitigation strategies. Recently, Boris has been actively involved in industry discussions focusing on PK and immunogenicity assessment, bioanalytical support of various biotherapeutic modalities, including mAbs, bi-specific antibodies, antibody-drug conjugates, ADCs and gene therapy. Boris is proud to be an active member of the American Association of Pharma Scientists.

This interview was conducted by Sankeetha Nadarajah, Managing Commissioning Editor of Bioanalysis, at the AAPS ICH-M10 Public Consultation Workshop (Silver Spring, MD, USA), 11 June 2019.
\end{abstract}

Accepted for publication: 15 July 2019; Published online: 6 September 2019

Keywords: guidance $\bullet \mathrm{ICH}-\mathrm{M} 10 \bullet$ regulation

Why was there a need for the ICH-M10 guidance, when there are already BMV guidance available?

Currently, there are several bioanalytical guidance documents available, including those from the US FDA, EMA, Canada, China, Japan and Brazil. Laboratories working across the globe are faced with the need to follow many, if not all, of these sets of recommendations that are in general quite similar although do contain some different expectations. Large pharmaceutical and biotech companies, like the one I work at, often submit applications for biological and chemical license approvals with multiple regions, countries, and are expected to meet the requirements of all of the different guidance documents relevant to the submisson, including those related to the bioanalytical assays used in the process of compound development. This can be challenging and/or confusing. To resolve this concern, we very much welcome a harmonized global guidance document such as the ICH-M10 guidance.

How will the ICH-M10 guidance be different to currently available BMV guidance, especially the FDA guidance from last year?

The FDA BMV (bioanalytical method validation guidance) was released last year while being in the works for quite some time. A considerable and broad discussion process was required during draft guideline review process. I believe that the final product is quite good. But again, the BMV document represents interests and position of the FDA organization, while other regional or country specific guidance documents remain in place. Through the issuance of the ICH-M10, we should see many if not all of the differences addressed with ideally one document to follow going forward.

I also want to add my personal opinion. I think that the FDA guidance, as good as it is, still has some questions that remain to be addressed. We would like to resolve them and hopefully, the ICH-M10 guidance is going to do just that. 
Thinking ahead to the future, the field of the Bioanalysis continues to evolve. New technologies, analytical protocols and platforms are constantly produced and implemented which allows to support an evolving range of therapeutic modalities and applications. The ICH-M10 guidance hopefully should capture the spirit of always evolving science of bioanalysis.

\section{Are there any disadvantages of such a collective consensus guidance?}

I do not believe so. As I was already suggesting earlier, I think having a uniform guidance document will be only beneficial. There are some differences in how the term Guidance is perceived in different localities. In some countries, the upcoming ICH-M10 may be viewed as a true set of guidelines while others may treat it more strictly. The goal of many discussions that we continue to have across the industry with the participation of many regulators is to produce a document that is clear on the message, allows to align on the interpretation and expectations set forward for the bioanalytical laboratories. The clear benefit will be to have one single document versus many.

\section{How easily can laboratories adapt to implement the ICH-M10 guidance?}

I think a lot of the ideas that are in the guidance now have been in the works or have been discussed and aligned with, so it is a matter of making sure that we interpret the statements made within the ICH-M10 in the same way and follow. I do not believe that there is going to be a significant shift, although some changes will be required. Like I said earlier, some of the recommendations that appear within the ICH-M10 draft can already be found in the EMA, FDA and other guidance documents. There are also clearly new forward-looking ideas which are very much welcomed. I think that implementation of the ICH-M10 should not be a significant concern. There are some changes that are still in discussion and these will require some adjustments.

\section{Will the ICH-M10 guidance meet the expectation from industry on a harmonized consensus guidance?}

Yes, it can reach a consensus with the hope that the comments that the industry has been working hard to generate and is providing the ICH-M10 Expert Group are considered and hopefully addressed, which is always possible to a degree. You can only assume that certain comments will be incorporated although I'm sure there will be some of us who will be a little disappointed. In general, in my own opinion, the draft of the ICH-M10 guidance is a very well written document. I think it clarifies a lot of the concerns that we had in the past while also addressing forward-looking position on the new technologies and ideas that have been touched on in previous discussions between regulators and the industry. The ICH-M10 brings clarity to several points made in the FDA bioanalytical method validation (BMV) guidance that we have been concerned about. I think it makes a lot of people more comfortable with the expectations.

\section{How regularly should the finalized guidance be reviewed and updated?}

It can take many years to develop a high quality guidance document that both regulators and the industry can work with. It is a very extensive and laborious process. Clearly, we cannot expect that this document can be frequently updated and issued. The review involves a considerable effort by the regulatory colleagues as well as requires a significant commitment from the industry to review and provide comments. In general, I do not propose that guidance documents should be reviewed and updated frequently but I would like to separate the terms 'reviewed' and 'updated'. I believe all guidelines should be 'reviewed' on a frequent basis to ensure continuous understanding and alignment with the most current state of the scientific field. True updates should and will probably come on a much less frequent basis. The way I understand the term regulatory guidance is, it is not a 'law'. It is a document that sets forth a set of rules, principles and expectations for the specific field of science based on the regulatory agency understanding of the associated needs, requirements aiming to optimize quality of the final product. The guideline presents regulatory agency position, which may change as the science continues to evolve and advance further. Current version of the ICH-M10 clearly suggests that the industry should reach out should we have a question on a particular topic and seek an understanding with the regulators. I very much appreciate cooperative position of the regulators. That is why I am saying that if we separate 'reviewed' from 'updated', we can always look at the guidance as a set of ideas that are put forward by regulators while we can look at these ideas and ask whether we reconsider certain statement based on the newly available information and data. This approach opens the door for a discussion but does not require a regular update of the document. Updates will be required, but do not expect them to be happening within several years. 


\section{How will the ICH-M10 guidance change the bioanalytical regulatory landscape?}

The biggest change, of course, is the fact that we are going to have one document that governs the regulatory position on bioanalysis from many, many countries' point of view. That is a huge achievement and clearly a significant step forward. It allows all of us in the industry to work on trying to align with one document, not several. It also allows us to align on the interpretation with the hope that all of the geographical regions will view the language of the ICH-M10 document similarly. To me, this is the biggest achievement, and that is why the ICH-M10 is very much welcomed.

\section{Disclaimer}

The opinions expressed in this interview are those of the interviewee and do not necessarily reflect the views of Newlands Press Ltd.

Financial \& competing interests disclosure

B Gorovits is an employee of Pfizer Inc. that is involved in the development of biotherapeutics and is compensated for his work by the company. The interviewee has no other relevant affiliations or financial involvement with any organization or entity with a financial interest in or financial conflict with the subject matter or materials discussed in the manuscript apart from those disclosed. No writing assistance was utilized in the production of this manuscript. 